University of New Hampshire

University of New Hampshire Scholars' Repository

Space Science Center

Institute for the Study of Earth, Oceans, and

Space (EOS)

1997

\title{
The prompt cosmic-ray-induced background in the orbiting Compton telescope COMPTEL
}

James M. Ryan

University of New Hampshire, James.Ryan@unh.edu

S C. Kappadath

University of New Hampshire - Main Campus

Mark L. McConnell

University of New Hampshire - Main Campus, mark.mcconnell@unh.edu

D Morris

University of New Hampshire - Main Campus

V Schonfelder

Max-Planck-Institut für extraterrestrische Physik

See next page for additional authors

Follow this and additional works at: https://scholars.unh.edu/ssc

Part of the Astrophysics and Astronomy Commons

\section{Recommended Citation}

Ryan, J.M.; Kappadath, S.; McConnell, M.; Morris, D.; Schonfelder, V.; Varendorff, M.; Weidenspointner, G.; Hermsen, W.; Bennett, K., "The prompt cosmic-ray-induced background in the orbiting Compton telescope COMPTEL," High Energy Radiation Background in Space, 1997 Conference on the , vol., no., pp.13,16, 22-23 Jul 1997

This Conference Proceeding is brought to you for free and open access by the Institute for the Study of Earth, Oceans, and Space (EOS) at University of New Hampshire Scholars' Repository. It has been accepted for inclusion in Space Science Center by an authorized administrator of University of New Hampshire Scholars' Repository. For more information, please contact Scholarly.Communication@unh.edu. 


\section{Authors}

James M. Ryan, S C. Kappadath, Mark L. McConnell, D Morris, V Schonfelder, M Varendorff, G Weidenspointer, W Hermsen, and K Bennett 


\title{
The Prompt Cosmic-Ray-Induced Background in the Orbiting Compton Telescope COMPTEL
}

\author{
J.M. Ryan, S. Kappadath, M. McConnell, D. Morris \\ University of New Hampshire, Durham, NH 03824, USA \\ V. Schönfelder, M. Varendorff, G. Weidenspointner \\ Max-Planck-Institut, 85740 Garching, Germany \\ W. Hermsen \\ SRON-Utrecht, 3484 CA Utrecht, The Netherlands \\ K. Bennett \\ ESA/ESTEC, 2200 AG Noordwijk, The Netherlands*
}

\begin{abstract}
We report the spectrum of background events that the COMPTEL instrument on the Compton Gamma Ray Observatory experiences due to the instantaneois effects of cosmic rays. Other backgrounds are present in the data of this Compton telescope, but the components that closely follow the instantaneous flux of cosmic rays are the most identifiable. The background varies approximately linearly with the cosmic-ray intensity and exhibits a broad feature from about 1 to $10 \mathrm{MeV}$ suggestive of the nuclear nature of the events. Above $10 \mathrm{MeV}$ there is a marked change in the nature of the background that is not understood. It appears that the background changes from nuclear to electromagnetic in nature.

\section{INTRODUCTION}

One of the major objectives of the COMPTEL experiment on the Compton Gamma Ray Observatory is the measurement of the cosmic diffuse gamma-ray flux [1]. Oddly enough, one of the advantages enjoyed by COMPTEL is that it experiences a varying background environment. The varying nature of the background is essential for measuring the cosmic diffuse flux [2]. As it orbits the Earth on the Compton Observatory, COMPTEL often views (toward the local zenith) the high galactic latitude portions of the sky, free from terrestrial gamma radiation. It is these data, away from the Galactic plane and away from the Earth, that we use to measure the cosrnic diffuse gamma ray flux. However, these data still contain significant amounts of background that must be correctly estimated to derive a cosmic diffuse spectrum.

The operation of COMPTEL has been described by Schönfelder et al. and the instrument is shown schematically in Fig. 1 [3]. COMPTEL is a double-scatter gamma-ray telescope/spectrometer. Its energy range is $0.8-30 \mathrm{MeV}$ and it has a field-of-view of approximately 1 sr. It consists of two independent ganma-ray detector subsystems, D1 and D2.
A gamma ray scatters in D1, the forward low-Z (NE213A) scintillation detector. It scatters again in D2, the rearward high- $\mathrm{Z}(\mathrm{NaI})$ scintillation detector. The energy deposit in both detectors and the time of flight (TOF) between the detectors is measured (as is the pulse shape in D1 to reject many neutron-generated events.) The event is accepted if both scintillations are above threshold and occur within $\sim 5 \mathrm{~ns}$ of one another, with the D1 signal occurring first. Additionally, no signal from the surrounding charged particle shields is allowed within $\sim 100$ ns of the coincidence.

The background experienced by COMPTEL that affects our ability to conduct measurements consists of two major components. There is first a background component that comes from the activation of radioactive isotopes. Most of the activation is created as the instrument passes through the South Atlantic Anomaly, the lowest altitude part of the Earth's radiation belts. This background builds up and decays depending on the instrument's exposure to the belts and the lifetime of the particular isotope. The other component, the focus of this discussion, is a prompt background that closely follows the instantaneous and local cosmic-ray intensity.

Cosmic-ray particles continuously bombard both the instrument and the spacecraft. For the most part these take the form of primary protons or secondary neutrons from the Earth's atmosphere. Multiple gamma rays are generated within and around the instrument that "simultaneously" interact with the independent detectors that constitute the COMPTEL instrument. These coincident gamma-ray interactions in the independent detectors of COMPTEL give rise to events that pass all the logic and electronic criteria for a legitimate gamma-ray event. The multiple gamma-ray emissions that constitute the prompt background fall into two categories. The first is the photons that come from cascading de-excitation of nuclei excited by cosmic rays. In this case nuclei are excited into high quantum states (or the continuum) and they de-excite by multiple photon emission on very short time scales. The photon emission from this process should closely track the local and instantaneous cosmic-ray intensity This work was supported by NASA contract NAS5-26645, by the German government through DARA grant 50 QV90968 and by the Netherlands
Organization for Scientific Research (NWO). 
and should be at energies corresponding to nuclear levels, i.e., up to and perhaps beyond $8 \mathrm{MeV}$. Photon showers from a deexciting nucleus can therefore produce acceptable signals in the instrument with a broad range of TOF values (from D1 to D2) depending upon the location of the excited nucleus (local mass distribution) relative to the individual detectors. The radiating nucleus, if it is within the instrument, must be excited by a fast neutron since proton-induced events are rejected by the charged-particle shields. If a neutron interaction occurs in the mass directly associated with the D1 detectors the consequent "gamma-ray" event will have the correct TOF to be accepted as a cosmic photon. Fig. 2 shows the distribution of TOF values from high-galactic-latitude observations in the energy range of $10-30 \mathrm{MeV}$, above the nuclear cascade range. The gaussian peak corresponds to the correct gamma-ray TOF from D1 and D2. It contains not only good cosmic photons but also local cascade events produced around the D1 detectors. The underlying continuum contains contributions from chance coincidences (flat baseline) and from other cascade events (declining curve) that are broadly distributed in TOF. The chance coincidences underlie the other components in the TOF spectrum. The shape of the declining continuum derives from the physical distribution of matter around COMPTEL. This includes the other instruments on the Compton Observatory that serve as sites of photon showers from primary cosmic-ray protons.

Activation-based events occur at the proper "gamma-ray" TOF and below. The cosmic diffuse flux is entirely restricted to the gaussian peak.

Electromagnetic showers are the second origin of multiple photon events. They are also present in the space environment. These can arise from primary cosmic-ray electrons or from photons or electrons produced in nearby nuclear cascades. Pions produced in nuclear cascades generate photons and electrons. The photons from these showers can extend well beyond the "nuclear" upper limit of several $\mathrm{MeV}$.

Both prompt TOF components are assumed to vanish in a zero cosmic ray environment.

The COMPTEL orbit nominally at $450 \mathrm{~km}$ takes it up to $28.5^{\circ}$ in latitude, both north and south. This path covers a wide range of geomagnetic cosmic-ray environments. The geomagnetic vertical cutoff rigidity varies from approximately 5 to $15 \mathrm{GV}$. This varying environment is reflected in the count rates of the four charged-particle shields that surround both major subsystems of COMPTEL. The shields function to identify the numerous incident charged particles, mainly protons above several $\mathrm{GeV}$. By the processes described above these protons give rise to showers rich in gamma rays, so they must be rejected. The so-called "veto" scalar rate (or deadtime clock counts) of one of these detectors, V2, varies from $\sim 2500$ to $\sim 650$ counts $/ 2.048 \mathrm{~s}$ at the extremes of the cosmic-ray environment. As we will show below, the frequency of prompt cosmic-ray proton-induced legitimate gamma-ray events registered in COMPTEL varies approximately linearly with this veto scalar rate.

One of the most powerful tools that one has with Compton telescopes is the TOF measurement. It represents the time separation of the signals between the D1 detector and the $\mathrm{D} 2$ detector. A true gamma ray within the aperture of the instrument scattering in the two detectors will have a $5 \mathrm{~ns}$ TOF value representing the $1.5 \mathrm{~m}$ separation of the two detectors. Shower or multiple gamma rays from outside the aperture will have other TOF values depending on the point of origin of the photons relative to the positions of the detectors. Multiple gamma rays generated in and around the D1 detector subsystem will have TOF values slightly less than the nominal $5 \mathrm{~ns}$ value. The TOF spectrum from a selected data set is shown in Fig. 2. The peak at channel 120 consists of events of cosmic origin and events generated in and around D1. The continuum beneath the peak is due to showering from other parts of the instrument and spacecraft and from chance coincidences. The spectrum in the figure has been decomposed into three components: the gaussian peak at channel 120 , the continuum, and a constant component, related to the chance coincidence events.

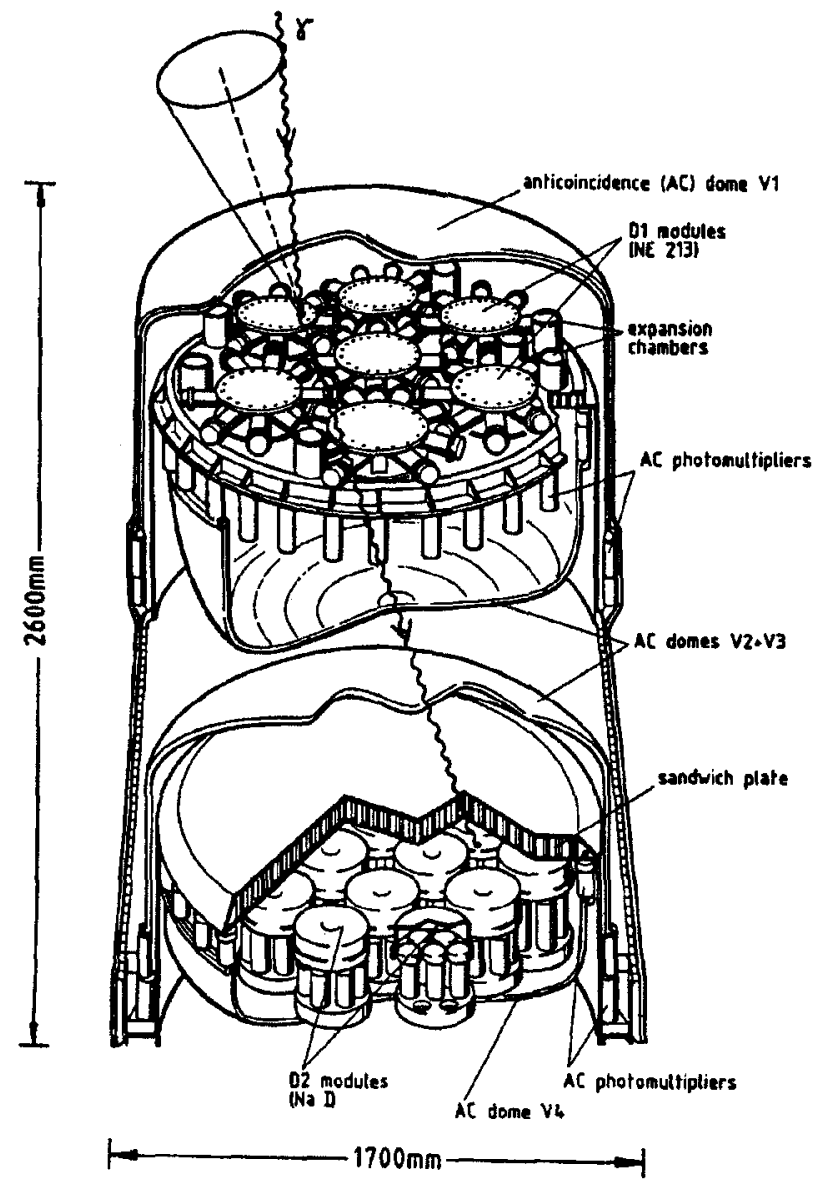

Fig. 1. Schematic drawing of COMPTEL.

\section{MEASUREMENTS}

In the study of the cosmic diffuse gamma-ray flux, many weeks of data were accumulated from pointings toward Virgo, well away from the Galactic plane. From these data a subset was selected from periods when the spacecraft was pointed near the zenith (no more than once per orbit). Additionally, gamma rays that had possible trajectories near the edge of the 
field-of-view and that could have originated from the Earth's atmosphere were rejected. This highly culled data set was studied for its background signal and subsequently its signal from the cosmic diffuse flux.

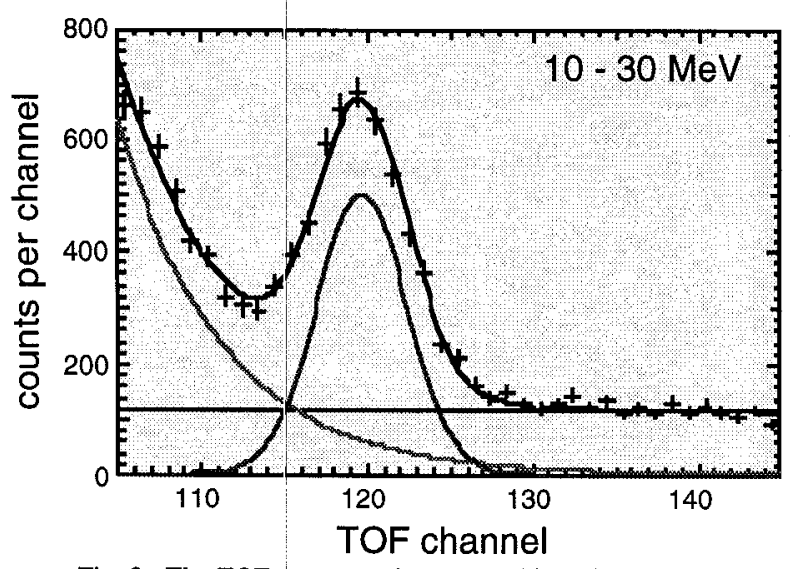

Fig. 2. The TOF spectrum decomposed into three components.

The amplitude of each component of the TOF spectrum can be correlated with the intensity of the local cosmic-ray flux as measured with the veto detectors. The veto system for COMPTEL consists of four independent detectors. The amplitude of the gaussian peak in Fig. 2 is plotted against the count rates of each of the veto detectors to search for any systematic differences in the measurements of the local cosmic-ray flux. This is shown in Fig. 3. The rates in veto detectors V1 and V3 are indistinguishable in the figure. (The intercept at the vertical axis is the basis for determining the cosmic diffuse gamrha-ray flux in that energy interval.) The different sizes and locations of the veto detectors are reflected in the different count rates, but all show linear behavior with no obvious signs of systematic non-linearities.

For the purpose of measuring the cosmic diffuse flux, these rates are extrapolated to zero. However, since the long-lived activation background (below $\sim 4 \mathrm{MeV}$ ) is unrelated to the instantaneous cosmic-ray flux, in the cosmic diffuse gammaray flux analysis these effects are first subtracted before fitting the prompt instrument background to the veto rate. The slopes of these curves in different energy ranges is a measure of the energy-dependent rate of multiple-photon cascade events that Compton telescopes suffer in space. Eventually, they constitute the background that limits the sensitivity of such instruments.

The gaussian peak sits atop the TOF continuum and a plot similar to Fig. 3 can be constructed for the intensity of the continuum as a function of cosmic-ray intensity. For any given energy interval, although not shown, the behavior of the TOF continuum is also linear, once the effect of longlived radioactive isotopes is removed. The linear dependence of the rate in a given energy interval is attributed to the prompt effect of cosmic rays.

\section{RESULTS AND DISCUSSION}

We selected energy intervals for the cosmic diffuse flux study based upon the nature of the background in that energy range. This results in energy intervals in the low range that encompass strong long-lived activation lines, e.g., ${ }^{24} \mathrm{Na},{ }^{22} \mathrm{Na}$. We restrict our study to the energy intervals of 1.8-2.7, 2.7$4.2,4.2-9,9-12,12-17$ and $17-30 \mathrm{MeV}$. The higher energy ranges are chosen to more evenly distribute statistics even though no long-lived activation lines are present above 4 MeV. Fig. 4 shows several count-rate spectra. The count rates that are plotted have been scaled by the COMPTEL efficiency at the appropriate energy yielding a shape that one can compare to a photon spectrum. The rates have also been evaluated at a veto count rate of $1000 \mathrm{~Hz}$, equivalent to a vertical rigidity cutoff of $\sim 5 \mathrm{GV}$, similar to the conditions at Palestine Texas. These include (1) the count rate spectrum of the prompt component in the gaussian peak, (2) the count rate spectrum of the TOF-continuum component in a $5 \mathrm{~ns}$ TOF window beneath the gaussian peak and (3) an $E^{-2.1}$ power law spectrum for reference to show the general shape of the cosmic diffuse spectrum, the signal against which the background must be compared [4].

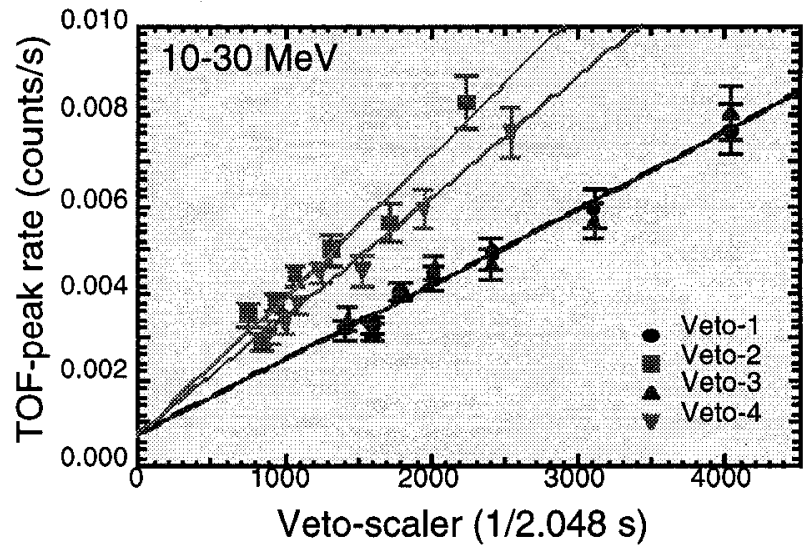

Fig. 3. The amplitude of the gaussian TOF peak as a function of cosmicray rate as measured with four charged-particle detectors.

The gaussian peak component above $1.8 \mathrm{MeV}$ has a hard spectral shape relative to that of the continuum. The gaussian component points below $1.8 \mathrm{MeV}$ are only rough upper limits and could be larger. (The rate in the gaussian peak in this range shows little variation with the cosmic-ray intensity.) However, the important point is that the continuum background dominates below $\sim 1.8 \mathrm{MeV}$. From about 1.8 to about $9 \mathrm{MeV}$, the two components are of comparable intensity with the gaussian component somewhat larger. Above $9 \mathrm{MeV}$ the gaussian peak component is the dominant background component. It appears to be increasing in relative importance as energy increases. The continuum component continues downward steeply after $10 \mathrm{MeV}$, suggestive of a nuclear origin. If this is so, then we can speculate that the gaussian component above $10 \mathrm{MeV}$ must be electromagnetic in origin if, like the continuum component, the nuclear component falls off at $\sim 10 \mathrm{MeV}$. One would expect then that below $9 \mathrm{MeV}$ the gaussian component consists of both a nuclear and an electromagnetic component since the two components are comparable below 9 $\mathrm{MeV}$. 
The prompt background in the gaussian peak has an intense and broad enhancement from approximately 2 to $8 \mathrm{MeV}$. This feature, found in earlier reports of cosmic diffuse flux spectrum, is variable in nature, depending only on the local cosmic-ray environment [5-7]. Its variability is seen in the COMPTEL data and can now be removed [2].

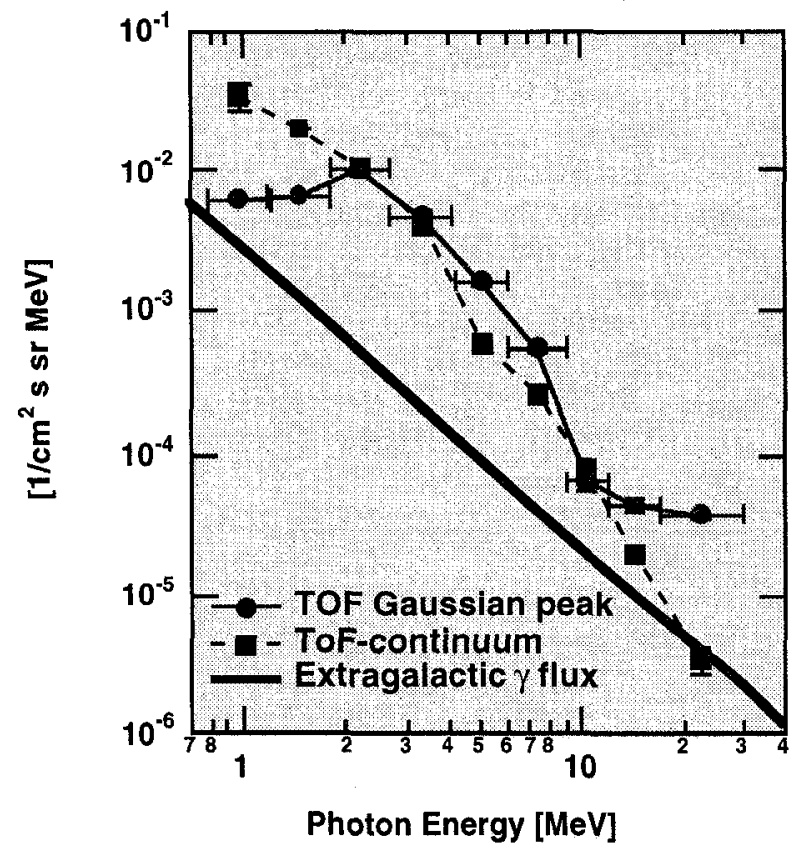

Fig. 4. Count rate spectra for different background components with the cosmic-diffuse reference power law [4]. The error bars are smaller than the symbols representing the data.

\section{CONCLUSIONS}

The prompt background of gamma rays experienced by COMPTEL exhibits at least two components: one from material in the aperture of the telescope and the other from material in other surrounding material. They can be distinguished from one another by their distributions in TOF. Within the gaussian peak background component there is evidence of two forms of background that become apparent as one progresses from below to above $10 \mathrm{MeV}$. Further study is required to identify these components in greater detail. These studies are warranted because they can affect the design of future instruments. For example, different materials, e.g., high $\mathrm{Z}$ materials, may be more efficient at producing cascade gamma rays and should be avoided if possible in designs of future Compton telescopes.

The background components discussed here must be added to the effects of the buildup of radioactive isotopes in the instrument. In measuring the cosmic diffuse gamma-ray flux, these isotopes can be more problematic than the prompt components presented here. Work is in progress to identify and quantify the activity of the isotopes that contribute heavily to the background below $4 \mathrm{MeV}$.

\section{REFERENCES}

[1] J. M. Ryan, "The scientific objectives of COMPTEL on the Gamma Ray Observatory," Nucl. Phys. B, vol. 10B, pp. 121-129, 1989.

[2] S. C. Kappadath, et al., "The preliminary cosmic diffuse gamma-ray spectrum from $800 \mathrm{keV}$ to $30 \mathrm{MeV}$ measured with COMPTEL," Bull. Amer. Astron. Soc., vol. 187, 1995.

[3] V. Schönfelder, et al., "Instrument description and performance of the imaging gamma-ray telescope COMPTEL aboard the Compton Gamma-Ray Observatory," Astrophys. J. Supp. Ser., vol. 86, pp. 657692, 1993.

[4] P. Sreekumar, et al., Astrophys. J., in press, 1997.

[5] R. S. White, B. Dayton, S. Moon, J. M. Ryan, R. B. Wilson, and A. D. Zych, "Cosmic diffuse gamma rays from 2 to $25 \mathrm{MeV}$," Astrophys. J., vol. 218 , pp. 920-927, 1977

[6] J. I. Trombka, C. Dyer, L. G. Evans, M. J. Bielefeld, S. M. Seltzer, and A. E. Metzger, "Reanalysis of the Apollo cosmic gamma-ray spectrum in the 0.3 to $10 \mathrm{MeV}$ energy region," Astrophys. J., vol. 212, pp. 925$935,1977$.

[7] V. Schönfelder, F. Graml, and E.-P. Penningsfeld, "The vertical component of 1-20 MeV gamma rays at balloon altitudes," Astrophys. $J .$, vol, 240, pp. 350-362, 1980. 